\title{
Structural studies of West Nile virus in complex with neutralizing antibodies
}

Richard J Kuhn*1, Bärbel Kaufmann'1, Shee Mei Lok ${ }^{1}$ Mick Cherrier ${ }^{1}$, Rushika Perera ${ }^{1}$, Dagmar Sedlak1 ${ }^{1}$ Grant E Nybakken ${ }^{2}$, Paul R Chipman ${ }^{1}$, Wei Zhang ${ }^{1}$, Michael S Diamond ${ }^{2}$, Daved H Fremont ${ }^{2}$ and Michael G Rossmann ${ }^{1}$

Address: ${ }^{1}$ Department of Biological Sciences, Purdue University, West Lafayette, IN 47907, USA and ${ }^{2}$ Department of Pathology and Immunology, Washington University School of Medicine, St. Louis, MO 63110, USA

Email: Richard J Kuhn* - kuhnr@purdue.edu

* Corresponding author

from Infectious diseases of the nervous system: pathogenesis and worldwide impact

Paris, France. 10-13 September 2008

Published: 23 September 2008

BMC Proceedings 2008, 2(Suppl I):S25

This abstract is available from: http://www.biomedcentral.com/I753-656I/2/SI/S25

(C) 2008 Kuhn et al; licensee BioMed Central Ltd.

West Nile virus (WNV) is a positive strand RNA virus in the family Flaviviridae, which includes members such as dengue, Japanese encephalitis, tick-borne encephalitis, yellow fever and Hepatitis C. As with other members of the genus, it is arthropod transmitted and has recently established itself as an endemic virus in the United States. Although most infections are asymptomatic, clinical manifestations of WNV include encephalitis and death. We have been interested in investigating the nature of the immune response with particular emphasis on the role of antibodies in reducing the level of infection. We have used a combination of techniques, but primarily structure, as a tool to probe the nature of antibody-mediated virus neutralization. Our results suggest that neutralization of virus particles is more complex than originally envisioned, with multiple mechanisms utilized. Using a combination of Xray crystallography and cryo-electron microscopy, several virus-antibody complexes have been determined at pseudo-atomic resolution. These studies suggest the following: 1) flavivirus particles exhibit dynamic motions or breathing that transiently expose cryptic epitopes; 2) although there are 180 potential binding sites for each monoclonal antibody the quasi-equivalent nature of the virion usually permits only a subset of sites to be utilized; 3 ) the availability of these sites, the epitope itself, and the avidity of antibody directly influence the mechanism of neutralization; and 4) particles thought to be incapable of infecting cells, so-called immature viruses, may play a critical role in immune surveillance and reactivity. The structure of the flavivirus virion and complexes of monoclonal antibodies will be presented along with data to support mechanisms antibody-mediated flavivirus neutralization. 